\title{
PRODUCT OF PROJECTIVE REPRESENTATIONS IN DESCRIPTION OF MULTI-ELECTRON STATES IN AN EXTERNAL MAGNETIC FIELD
}

\author{
W. FLOREK* \\ Computational Physics Division, Institute of Physics \\ Adam Mickiewicz University \\ Umultowska 85, 61-614 Poznań, Poland
}

(Received October 27, 1998; revised version February 1, 1999)

\begin{abstract}
In this paper all inequivalent irreducible projective representations of the two-dimensional translation group for a given factor system are determined. A normalized, i.e. corresponding to the Landau gauge, factor system is considered. Obtained representations directly lead to concept of magnetic cells and to periodicity with respect to the charge of a moving particle. It is also shown that the quantization condition is imposed on the product $q H$ of the charge $q$ and the magnitude of magnetic field $H$. The Kronecker product of such representations is considered and it is proven that the multiplication of representations corresponds to the addition of charges of particles moving in a given external magnetic field. In general, coupling of $d$ representations corresponds to $d$-particle states. Presented results can be applied in any problem related to two-dimensional electron gas in a magnetic field, for example in the fractional quantum Hall effect or high temperature superconductivity.

PACS numbers: 03.65.Fd, 02.10.Sp, 71.70.Di
\end{abstract}

\section{Introduction}

Projective representations investigated by Schur [1] at the beginning of this century are widely applied in quantum mechanics, since their factor systems are related to the freedom in choosing phase factors of state vectors (see, e.g., Altmamn [2]). In the sixties Brown [3] applied them in investigation of the movement of a Bloch electron in a magnetic field. Almost at the same time Zak [4] proposed an equivalent approach: projective representations of the translation group were considered as vector representations of its covering group, being in fact a central

*e-mail: florek@amu.edu.pl 
extension of the translation group and a group of factors (see also [5]). The problem investigated by these authors is strongly related to the Landau quantization [6] and, therefore, to the quantum Hall effect $[7,8]$.

In the previous papers $[5,9]$ the magnetic translation group was studied within the frame proposed by Zak, i.e. as a central extension of the translation group. Similar approach was also proposed by Divakaran and Rajagopal [10]. These investigations gave a basis to construct and take into account all representations, including those con:idered previously by Zak [4] as "non-physical" [11]. Moreover, the physical relevance and possible applications of such representations were indicated. In this article the magnetic translation operators are studied applying Brown's approach, i.e. projective representations of the two-dimensional translation group are considered.

In Sec. 2, after recalling Brown's definitions, matrix elements of finite irreducible projective representations are given. They are obtained from those introduced by Brown by application of the appropriate gauge transformation. In the next section the tensor (Kronecker) products of such representations are investigated. Starting from a simple example physical relevance of the structures considered is discussed in Sec. 4. Periodicity of representations (therefore also physical properties) with respect to charge of a moving particle is shown. The last section contains an overall summary and final conclusions.

\section{Irreducible projective representations}

Brown [3] introduced magnetic translations as unitary operators determined for a crystal lattice vector $\boldsymbol{R}$ and a vector potential $\boldsymbol{A}$ as

$$
T(\boldsymbol{R})=\exp \left[-\frac{\mathrm{i}}{\hbar} \boldsymbol{R} \cdot\left(p-\frac{e}{c} \boldsymbol{A}\right)\right]
$$

where $p$ is a linetic momentum. These operators commute with the well-known Hamiltonian

$$
\mathcal{H}=\frac{1}{2 m}\left(p+\frac{e}{c} \boldsymbol{A}\right)^{2}+V(r)
$$

describing an electron in a periodic potential $V(r)$ and a uniform, constant magnetic field

$$
\boldsymbol{H}=\operatorname{rot} \boldsymbol{A}, \quad \text { where } \quad \boldsymbol{A}=\frac{1}{2}(\boldsymbol{H} \times r) .
$$

The above defined operators form a projective representation of the crystal translation groups, which is expressed by the following relation:

$$
T(\boldsymbol{R}) T\left(\boldsymbol{R}^{\prime}\right)=T\left(\boldsymbol{R}+\boldsymbol{R}^{\prime}\right) m\left(\boldsymbol{R}, \boldsymbol{R}^{\prime}\right)
$$

with

$$
m\left(\boldsymbol{R}, \boldsymbol{R}^{\prime}\right)=\exp \left[-\frac{1}{2} \mathrm{i}\left(\boldsymbol{R} \times \boldsymbol{R}^{\prime}\right) \cdot h\right]
$$

being a factor system of this representation and $h=e \boldsymbol{H} / \hbar c$. Imposing the periodic boundary conditions Brown [3] has shown (see also [4]) that the magnetic field has to be quantized, satisfying the following condition:

$$
h=\frac{2 \pi}{N} \frac{a_{3}}{\Omega} L
$$


for an integer $1 \leq L<N$ mutually prime with the period $N$ of the crystal lattice ( $\Omega=\left(a_{1} \times a_{2}\right) \cdot a_{3}$ is the volume of the primitive cell and $a_{i}$ are the primitive translations). Condition (3) allows us to put $\boldsymbol{H}=H \hat{\boldsymbol{a}}_{3}$, where $\hat{\boldsymbol{a}}_{3}=a_{3} / a_{3}$. For the salke of simplicity we assume that $a_{3} / \Omega=1$ so (2) can be rewritten as

$$
\frac{e}{\hbar c} H=\frac{2 \pi}{N} L \quad \text { or } \quad H=\frac{2 \pi}{N} \frac{\hbar c}{e} L .
$$

For $h$ given by (2) and the factor system (1) Brown obtained $N$-dimensional irreducible projective representations for $\boldsymbol{R}=n_{1} a_{1}+n_{2} a_{2}$ with matrix elements given as

$$
D_{j k}(\boldsymbol{R})=\exp \left[\frac{\pi \mathrm{i}}{N} L n_{1}\left(n_{2}+2 j\right)\right] \delta_{j, k-n_{2}} \quad(\bmod N),
$$

where $j, k=0,1, \ldots, N-1$. The factor system for this representation agrees with that given by (1) since

$$
D(\boldsymbol{R}) D\left(\boldsymbol{R}^{\prime}\right)=D\left(\boldsymbol{R}+\boldsymbol{R}^{\prime}\right) \exp \left[\frac{\pi \mathrm{i}}{N} L\left(n_{2} n_{1}^{\prime}-n_{1} n_{2}^{\prime}\right)\right] .
$$

Note that all factors are roots of 1 of the order $2 N$, whereas the dimension of the representations considered is $N$. Therefore, this system is not normalized [2]. However, an equivalent normalized factor system, i.e. such a system that all factors are the $N$-th roots of 1 , can be obtained [12]. To normalize it the matrix $D(\boldsymbol{R})$ is multiplied by $\phi(\boldsymbol{R})=\exp \left(-\pi \mathrm{i} L n_{1} n_{2} / N\right)$. In this way a new irreducible representation can be written in the following form:

$$
\langle N, L ; 0\rangle_{j k}\left[n_{1}, n_{2}\right]=\delta_{j, k-n_{2}} \omega_{N}^{L n_{1} j}, \quad j, k=0,1, \ldots, N-1,
$$

where $\omega_{N}=\exp (2 \pi \mathrm{i} / N)$, the $\langle N, L ; 0\rangle$ denotes the representation (the role of the zero vector 0 will be explained below) and a vector $n_{1} a_{1}+n_{2} a_{2}$ is replaced by a pair $\left[n_{1}, n_{2}\right] \in Z_{N} \times Z_{N}$. Since only the unique element $[0,0]$ has nonzero character, then this representation is irreducible, a projective representation of the translation group $T_{N} \simeq Z_{N}^{2}$. It is easily shown that a factor system

$$
m_{N}^{(L)}\left(\left[n_{1}, n_{2}\right],\left[n_{1}^{\prime}, n_{2}^{\prime}\right]\right)=\omega_{N}^{L n_{2} n_{1}^{\prime}}
$$

corresponds to the representations given by $(5)$. It can be proven $[12,13]$ that this factor system corresponds to the Landau gauge, used in many papers $[8,14]$. Discussion on a form of vector potential and gauge invariance lies beyond the scope of this work and it is presented elsewhere [12]. Three important facts, however, should be stressed:

- Projective representations with different, though equivalent, factor systems are inequivalent $[2,15]$, therefore representations (4) and (5) are inequivalent. However, in both cases one can use the same set of basis functions.

- In fact, a modification of the factor system (1) corresponds to a different choice of the gauge $\boldsymbol{A}$; it was shown [13] how to introduce magnetic translations for any gauge $\boldsymbol{A}$ such that $\boldsymbol{H}=\operatorname{rot} \boldsymbol{A}$ and $\operatorname{div} \boldsymbol{A}=0$.

- Equivalent factor systems lead to the same expression for the commutator [12]

$$
D(\boldsymbol{R}) D\left(\boldsymbol{R}^{\prime}\right) D^{-1}(\boldsymbol{R}) D^{-1}\left(\boldsymbol{R}^{\prime}\right)=\omega_{N}^{-L\left(n_{1} n_{2}^{\prime}-n_{2} n_{1}^{\prime}\right)} .
$$


The actual form of basis function is not discussed here (see, e.g., $[3,4,6,16]$ for more details). These functions, denoted as $|s\rangle$ with $s=0,1, \ldots, N-1$, are eigenfunctions of $\langle N, L ; 0\rangle\left[n_{1}, 0\right]$ operators, whereas the operators $\langle N, L ; 0\rangle\left[0, n_{2}\right]$ permute them in a cyclic way (cf. $[5,17]$ )

$$
\begin{aligned}
& \langle N, L ; 0\rangle\left[n_{1}, 0\right]|s\rangle=\omega_{N}^{L n_{1} s}|s\rangle, \\
& \langle N, L ; 0\rangle\left[0, n_{2}\right]|s\rangle=\left|s-n_{2}\right\rangle \quad(\bmod N) .
\end{aligned}
$$

The special choice of $\langle N, L ; 0\rangle$ puts $a_{1}=[1.0]$ and $a_{2}=[0,1]$ on a different footing.

Let us assume that the number $L$ has a common factor with $N$, say $L=l \nu$ and $N=n \nu$ with $\nu=\operatorname{gcd}(L, N)>1$. Recall that (2) relates $L$ with the magnetic field magnitude. It is easy to notice that the magnetic periodicity is obtained for the smaller period $n$ and the factor system (6) can be written as

$$
m_{n}^{(l)}\left(\left[n_{1}, n_{2}\right],\left[n_{1}^{\prime}, n_{2}^{\prime}\right]\right)=\omega_{n}^{l n_{2} n_{1}^{\prime}} .
$$

Therefore, one may consider factor systems (8) for all divisors $n$ of $N$ and $l$ mutually prime with $n$ (i.e. $\operatorname{gcd}(n, N)=n$ and $\operatorname{gcd}(l, n)=1$ ). It is an easy task of combinatorics (see, for example, a book by Kerber [18]) to show that $N$ different factor systems, corresponding to $L=0,1, \ldots, N-1$, are obtained in this way. Since even for $n<N$ the $N \times N$ crystal lattice is considered, so $N$ will be called hereafter the crystal period, whereas $n$, for which $T(n \boldsymbol{R})=\mathbf{1}$, will be called the magnetic period. Hence, the $N \times N$ lattice can be viewed as a $\nu \times \nu$ lattice, with the translation group $T_{\nu}=Z_{\nu}^{2}$, of $n \times n$ magnetic cells. Let $k=\left[k_{1}, k_{2}\right]$ and

$$
\langle k\rangle_{\nu}\left[\xi_{1}, \xi_{2}\right]=\exp \left[-2 \pi \mathrm{i}\left(k_{1} \xi_{1}+k_{2} \xi_{2}\right) / \nu\right]=\omega_{\nu}^{-\left(k_{1} \xi_{1}+k_{2} \xi_{2}\right)}
$$

be an irreducible representation of $T_{\nu}$. Then one can check that the $n$-dimensional matrices

$$
\langle n, l ; k\rangle_{j k}\left[n_{1}, n_{2}\right]=\delta_{j, k-\eta_{2}} \omega_{n}^{l \eta_{1} j} \omega_{\nu}^{-\left(k_{1} \xi_{1}+k_{2} \xi_{2}\right)}
$$

form a projective irreducible representation of the group $T_{N}$ with a factor system (8). In this formula $\left[\xi_{1}, \xi_{2}\right]$ labels magnetic cells, whereas $\left[\eta_{1}, \eta_{2}\right]$ labels positions within a magnetic cell, i.e. $n_{i}=\eta_{i}+\xi_{i} n$. As the basis function the eigenvectors $|n, l ; k ; s\rangle, 0 \leq s<n$, of the matrix $\langle n, l ; k\rangle[1,0]$ can be used. The character of the representation (10) is easily calculated as

$$
\chi\langle n, l ; k\rangle\left[n_{1}, n_{2}\right]=\delta_{\eta_{1}, 0} \delta_{\eta_{2}, 0} n \omega_{\nu}^{-\left\langle k_{1} \xi_{1}+k_{2} \xi_{2}\right)} .
$$

For given $n$ and $l$ (i.e. for a given factor system) we obtain $\nu^{2}$ inequivalent irreducible projective representations labelled by $k$, therefore we obtain all of them $[2,3]$. In particular, we have

$$
\langle 1,1 ; k\rangle=\langle k\rangle_{N} \text {. }
$$

\section{Products of irreducible projective representations}

It can be shown $[2,19]$ that a product of two projective representations $T$ and $T^{\prime}$ of a given group $G$ with factor systems $m$ and $m^{\prime}$ is another projective representation, denoted $T \otimes T^{\prime}$, with a factor system $m^{\prime \prime}\left(g, g^{\prime}\right)=m\left(g, g^{\prime}\right) \dot{m}^{\prime}\left(g, g^{\prime}\right)$, which, in general, is different from (and non-equivalent to) factor systems $m$ and $m^{\prime}$. In 
the case considered here all factors are the $N$-th root of 1 and a product of two factors $m_{n}^{(l)}$ and $m_{n^{\prime}}^{\left(l^{\prime}\right)}$ is equal to

$$
m\left(\left[n_{1}, n_{2}\right],\left[n_{1}^{\prime}, n_{2}^{\prime}\right]\right)=\omega_{N}^{\left(l \nu+l^{\prime} \nu^{\prime}\right) n_{2} n_{1}^{\prime}},
$$

therefore it corresponds to the representation with $L=l \nu+l^{\prime} \nu^{\prime}$. It means that the set of factor systems ( 8 ) is closed with respect to multiplication and, therefore, the representations (10) and their direct sums form a closed set with respect to the tensor product. Of course, we can add representations with the same factor system only and vice versa - if a given projective representation with a factor system $m$ is reducible then it can be decomposed into a direct sum of irreducible projective representations with the same factor system $m$. Moreover, the orthogonality relations for representations and their characters are valid for representations with the same factor system [2]. Hence, one must be careful decomposing a given projective representation, as representations with different factor systems cannot be compared.

Let $D$ be a product of two irreducible representations $\langle n, l ; k\rangle$ and $\left\langle n^{\prime}, l^{\prime} ; k^{\prime}\right\rangle$. Its factor system is given by (11), its dimension equals $n n^{\prime}$ and its character is

$$
\chi^{D}\left[n_{1}, n_{2}\right]=\delta_{\eta_{1}, 0} \delta_{\eta_{2}, 0} \delta_{\eta_{1}^{\prime}, 0} \delta_{\eta_{2}^{\prime}, 0} n n^{\prime} \omega_{N}^{-n\left(k_{1} \xi_{1}+k_{2} \xi_{2}\right)-n^{\prime}\left(k_{1}^{\prime} \xi_{1}^{\prime}+k_{2}^{\prime} \xi_{2}^{\prime}\right)},
$$

therefore it is nonzero only for $n_{i}=x_{i} m$, where $m=n n^{\prime} / \gamma, \gamma=\operatorname{gcd}\left(n, n^{\prime}\right)$, $0 \leq x_{i}<\mu=. V / m=\operatorname{gcd}\left(\nu, \nu^{\prime}\right)$. Substituting $m$ and $\mu$ to the above formula one easily obtains

$$
\chi^{D}\left[n_{1}, n_{2}\right]=\delta_{\eta_{1}, 0} \delta_{\eta_{2}, 0} m \gamma \omega_{\mu}^{-\left(k_{1}+k_{1}^{\prime}\right) x_{1}-\left(k_{2}+k_{2}^{\prime}\right) x_{2}} \quad(\bmod m) .
$$

Since $\nu / \mu=n^{\prime} / \gamma$ then $L$ in (11) can be written as

$$
L=\mu\left(\frac{l \nu}{\mu}+\frac{l^{\prime} \nu^{\prime}}{\mu}\right)=\mu\left(\frac{l n^{\prime}}{\gamma}+\frac{l^{\prime} n}{\gamma}\right)=\mu \Lambda .
$$

It seems that this determines a factor system $m_{m}^{(A)}$. However, we cannot exclude the case when $\operatorname{gcd}(\Lambda, m)=\ell>1$. It is evident that the summands in (13) have no common factor, but it may happen that their sum $\Lambda$ has a common factor with $m$. Therefore, the product considered has to be decomposed into irreducible representations with a factor system $m_{M}^{(\lambda)}$, where $\lambda=\Lambda / \ell$ and $M=m / \ell$. The scalar product of appropriate characters gives the frequency of the representation $\langle M, \lambda ; \kappa\rangle$ in the product of representations $\langle n, l ; k\rangle$ and $\left\langle n^{\prime}, l^{\prime} ; k^{\prime}\right\rangle$

$$
\begin{gathered}
f\left(\langle M, \lambda ; \kappa\rangle,\langle n, l ; k\rangle \otimes\left\langle n^{\prime}, l^{\prime} ; k^{\prime}\right\rangle\right)=\frac{m M \gamma}{N^{2}} \sum_{x_{1}, x_{2}=0}^{\mu-1} \omega_{\mu}^{-\left(k_{1}+k_{1}^{\prime}-k_{1}\right) x_{1}-\left(k_{2}+k_{2}^{\prime}-k_{2}\right) x_{2}} \\
=\frac{\gamma}{\ell} \delta_{k_{1}, k_{1}+k_{1}^{\prime}} \delta_{k_{2}, k_{2}+k_{2}^{\prime}} ;
\end{gathered}
$$

there are $\ell^{2}$ such representations with $\kappa_{i}=\left(k_{i}+k_{i}^{\prime}\right) \bmod \mu$.

The most interesting is the case when $n=n^{\prime}$ and $l=l^{\prime}$, since $n$ and $l$ are determined by the magnetic field magnitude (and the crystal period $N$ ). Therefore, representations $\langle n, l ; k\rangle$ and $\left\langle n, l ; k^{\prime}\right\rangle$ act in two $n$-dimensional eigenspaces of one-electron states and their product should correspond to two-electron space of 
states [11]. In this case one obtains that the resultant representation is $n^{2}$-dimensional and $\gamma=m=n, \mu=\nu$. From (12) the character is equal to

$$
\chi^{D}\left[n_{1}, n_{2}\right]=\delta_{n_{1}, x_{1} n} \delta_{n_{2}, x_{2} n} n^{2} \omega_{\nu}^{-\left(k_{1}+k_{1}^{\prime}\right) x_{1}-\left(k_{2}+k_{2}^{\prime}\right) x_{2}}
$$

with $0 \leq k_{i}, k_{i}^{\prime}, x_{i}<\nu$. The factor system is given by (11)

$$
m\left(\left[n_{1}, n_{2}\right],\left[n_{1}^{\prime}, n_{2}^{\prime}\right]\right)=\omega_{n}^{2 l n_{2} n_{1}^{\prime}}=\omega_{n}^{A n n_{2} n_{1}^{\prime}},
$$

where, see (13), $\Lambda=2 l$. At this moment the cases of odd and even $9 n$ have to be considered separately. In the first case $\ell=\operatorname{gcd}(n, 2 l)=1$ and the obtained representation decomposes into $n$ copies of the representation $\langle n, 2 l ; \kappa\rangle$ with $\kappa_{i}=$ $\left(k_{i}+k_{i}^{\prime}\right) \bmod \nu$. In the second case, however, $\ell=2$ and $M=\frac{1}{2} n$ therefore the considered product decomposes into representations $\left\langle\frac{1}{2} n, l ; \kappa\right\rangle$ : there are four representations with $\kappa_{i}-k_{i}-k_{i}^{\prime}=0, \nu$ and each of them appears $\frac{1}{2} n$ times. In a similar way, the coupling of $d$ representations $\left\langle n, 1 ; k^{(j)}\right\rangle, j=1,2, \ldots, d$ with $n=d M$ changes the magnetic period from $n$ to $M$, at the magnetic field kept constant the effective charge is multiplied by $d$.

Since the multiplicities of irreducible representations can be large [20] the Clebsch-Gordan coefficients for the product considered are strongly ambiguous and they are not discussed here.

\section{Periodicity with respect to charge}

Let us consider $N=12$ and two irreducible projective representations of $T_{12}=Z_{12}^{2}:\langle 3,1 ;[1,0]\rangle$ and $\langle 6,1 ;[1,0]\rangle$. Therefore, $\mu=2$ and, see (11), (13: $L=6=3 \mu$. Therefore, $\Lambda=3, \ell=\operatorname{gcd}(\Lambda, m)=3$ and the considered product decomposes into nine irreducible representations. To determine them one has to find that $M=m / \ell=2$ and $\lambda=A / \ell=1$. Therefore, a factor system of the resultant irreducible representations should be denoted as $m_{2}^{(1)}$ instead of $m_{6}^{(3)}$ and these representations are two-dimensional. Due to the condition $\kappa_{i}=\left(k_{i}+k_{i}^{\prime}\right)$ mod $\mu$ one obtains that $\kappa_{i}, i=1,2$, should be even. Since $N / M=6$ then $\kappa_{i}=$ $0,1,2,3,4,5$ and finally the following decomposition can be written:

$$
\langle 3,1 ;[1,0]\rangle \otimes\langle 6,1 ;[1,0]\rangle=\bigoplus_{\kappa_{1}, \kappa_{2}=0,2,4}\left\langle 2,1 ;\left[\kappa_{1}, \kappa_{2}\right]\right\rangle .
$$

To obtain the magnetic periodicity for $n=3, l=1$ and $n^{\prime}=6, l^{\prime}=1$ one has to assume that, according to Eq. (3) and the definition of magnetic periodicity below Eq. (8),

and

$$
H=\frac{2 \pi}{n} \frac{\hbar c}{e} l=\frac{2 \pi}{3} \frac{\hbar c}{e}
$$

$$
H^{\prime}=\frac{2 \pi}{n^{\prime}} \frac{\hbar c}{e} l^{\prime}=\frac{2 \pi}{6} \frac{\hbar c}{e} .
$$

It seems that there are two different magnitudes of the magnetic field since $H \neq H^{\prime}$. However, we can also assume that these representations correspond to two particles with charges $q e$ and $q^{\prime} e$, respectively $\left(q, q^{\prime}\right.$ are integers). In a general case of a particle carrying charge $q e$ the relative magnetic field amplitude has to be defined 
as $h=q e \boldsymbol{H} / \hbar c$. Since the condition (2) is imposed on $h$ not on $\boldsymbol{H}$, then, in a general case, the condition (3) has to be rewritten as

$$
H=\frac{2 \pi}{N} \frac{\hbar c}{e} \frac{L}{q} \text {. }
$$

Applying this definition we see that the magnetic periodicity can be obtained for both representations if charges of particles satisfy condition $q=2 q^{\prime}$, since in this case we have

$$
H=\frac{2 \pi}{3} \frac{\hbar c}{q e}=\frac{2 \pi}{6} \frac{\hbar c}{q^{\prime} e}=H^{\prime} .
$$

The same value of the magnetic field magnitude $H$ should be obtained for the resultant representations, corresponding to $M=2, \lambda=1$, therefore

$$
H^{\prime \prime}=\frac{2 \pi}{2} \frac{\hbar c}{q^{\prime \prime} e}=\frac{2 \pi}{6} \frac{\hbar c}{q^{\prime} e}
$$

and

$$
q^{\prime \prime}=3 q^{\prime}=q+q^{\prime} .
$$

In this way it has been shown that the multiplication of representations corresponds to addition of charges.

To determine a relation between the charge $q$ of a particle and the irreducible projective representation $\langle n, l ; k\rangle$ let us fix the magnetic field $H$ and the crystal period $N$. Then the condition (15) gives that $L \propto q$. However, it is not a one-to-one relation since $L$ is limited to the range $0,1, \ldots, N-1$ with no condition imposed on $q \in Z$. Let us assume that $H \neq 0$ has the smallest admissible value $H_{1}$ obtained from the conclition (15) for fixed $N$ (the crystal period) and $L=q=1$, i.e. $H_{1}=2 \pi \hbar c / N e$. The representation (5), its factor system (6), and the commutator (7) are determined by $\omega_{N}^{L}$, so all of them are periodic functions of $L \propto q$ and, therefore, periodic functions with respect to the charge of a moving particle. We see, in particular, that for $q=z N, z \in Z$, vector representations with trivial factor systems, and trivial commutators, are obtained. It means that for a given crystal period $N$ a particle with the charge $z N e$ behaves as non-charged one. We also notice that taking $q=1,2, \ldots, N-1$ we have to assume that $L=q$ (for the above defined smallest admissible $H$ ). Therefore, for some $q$ we can obtain $q=l \nu$, where $\nu=\operatorname{gcd}(N, q)$, and in this case the irreducible representations $\langle n, l ; k\rangle$ have to be used. Since $\nu$ is a co-divisor of $n$ then we finally obtain the following relation:

$$
q=l \nu=l \frac{N}{n}
$$

which relates the pair $(n, l)$ (a label of the irreducible representation) and the charge $q$ of a particle. It has to be stressed that this relation does not depend on the label $k$ of irreducible representations of $T_{\nu}$ of Eq. (9). Comparing relation (16) with Eqs. (11) and (13) one can see that addition of charges corresponds to multiplication of irreducible representations.

\section{Final remarks and conclusions}

It has been shown that all inequivalent projective representations of the 2D translation group with the Born-Kámann periodic boundary conditions form 
a closed set with respect to the Kronecker product. A general formula (14) for multiplicities of irreducible projective representations in a product of two others is given. It is important that the introduced irreducible representations (10) can be written as a product of a one-dimensional irreducible representation $\langle k\rangle$ of the translation group $T_{\nu}$ in Eq. (9) and a projective one of $T_{n}$. It means that also products of such representations can be separated into a part describing addition of the quasi-momenta (vectors in the reciprocal lattice) $k, k^{\prime}$ with the second part corresponding to the addition of co-divisors $\nu$ and $\nu^{\prime}$ or, more precisely, $l \nu+l \nu^{\prime}$, see Eq. (11) and below. However, the last addition can change the magnetic periodicity, determined by $M$ and $\lambda$ in Eqs. (13) and (14), in a way depending on the arithmetic structure of $N, n, n^{\prime}, l$ and $l^{\prime}$. In examples presented at the end of Sec. 3 and in Sec. 4 the label $M$ of resultant representation was equal to or was smaller than $n$ and $n^{\prime}$. It is easy to check that for $N=12, n=3, n^{\prime}=4$ and $l=l^{\prime}=1$ we obtain $M=12$ and $\lambda=7$, therefore $M>n, n^{\prime}$ and there is only one magnetic cell. Therefore, the addition of quasi-momenta $k, k^{\prime}$ has to be modified to reflect all possible changes of the magnetic periodicity.

Let us recall that projective representations are related with central extensions [2, 21, 19] which was, for example, exploited by Zak [4] in the theory of Bloch electrons or, in a general case by Divakaran [22] (this paper is accompanied by a review on central extensions written by Raghunathan [23]). Determination of central extensions by the Mac Lane method $[5,24]$ gives us all inequivalent ones. However, some of them can be isomorphic $[2,15]$ and from the mathematical point of view the obtained groups are identical. In the case of projective representations it means that mathematical description will be the same if a factor $\omega_{N}$ is replaced by $\omega_{N}^{r}$ for $r$ mutually prime with $N$, which corresponds to freedom in choosing relation between the charge $q$ and the index $l$ in Eq. (16). For $q=1$, i.e. considering one electron, we can take any $r$ mutually prime with $n$. All important physical properties, such as addition of charges and charge periodicity, are retrieved: since if $\operatorname{gcd}(r, n)=1$ then $\{r, 2 r, \ldots, n r\}=\{1,2, \ldots, n\}$, but elements of this set are obtained in different order (products $z r$ are calculated mod $n$ ). Therefore, observing only magnetic or charge periodicity wè cannot distinguish cases $H_{1}=2 \pi \hbar c / N e$ and $H_{r}=r H$ if $\operatorname{gcd}(r, N)=1$, see (15) and (16). On the other hand, it means that the condition (15) is not imposed on $H$ or $q$, but on their product $q H$.

Introduction of projective representations in this paper was based on the magnetic translation operators determined by Brown [3] and the notion of Bloch electrons in an external magnetic field was used throughout this work. Therefore, the concept of magnetic cells has appeared in a natural way. However, these representations can be applied to any problem in quantum mechanics, where a symmetry group $G$ appears and phase factors play important role. For example, Divakaran and Rajagopal [25] used them in the theory of superconducting layered materials, and they included also many general remarks in their publication. If we assume that projective representations correspond to energy levels (therefore representation vectors correspond to states) of a one-particle system then products of two (or more) representations have to correspond to two-particle (or many-particle, in a general case) systems. The simplest two-particle Hamiltonian commuting with the magnetic translations was presented and briefly discussed in the previous pa- 
per [11], therefore it has not been introduced in this paper. Non-trivial applications of the proposed approach are possible when a Hamiltonian contains pairwise iteractions. Not going far from physical problems discussed above we can look at two-dimensional electron gas in an external magnetic field. The fractional quantum Hall effect $[26,27]$ is still a subject of many efforts of theorists and experimentalists but it has been accepted that Coulomb interactions play a very important role in explanation of observed features [28]. Therefore, it seems possible to apply presented results to such problem. Moreover, since the condition (15) restricts the product $q H$, then it is evident that for high magnetic fields a fractional charge can be observed, if the product $q H$ is to satisfy (15) and (16).

\section{Acknowledgments}

It is a pleasure to thank Professor G. Kamieniarz for carefully reading a manuscript and many helpful remarks. A support from the Committee for Scientific Research within the project No. 2 P03B 02916 is acknowledged. The Stefan Batory Foundation supported participation of the author in the Fifth International Wigner Symposium, partial results of this work were presented at.

\section{References}

[1] I. Schur, J. Reine Angew. Math. 127, 20 (1904); ibid. 132, 85 (1907); Math. Ann. 71, 85 (1911).

[2] S.L. Altmann, Induced Representations in Crystal and Molecules, Academic Press, London 1977, Ch. 6; Rotations, Quaternions, and Double Groups, Clarendon, Oxford 1986, Ch. 8.

[3] E. Brown, Bull. Am. Phys. Soc. 8, 256 (1963); Phys. Rev. 133, A1038 (1964).

[4] J. Zak, Phys. Rev. 134, A1602, A160t (1964).

[5] W. Florek, Rep. Math. Phys. 34, 81 (1994).

[6] J. Zak, Phys. Rev. 136, A776, A1647 (1964).

[7] I. Dana, Y. Avron, J. Zak, J. Phys. C, Solid State Phys. 18, L679 (1985).

[8] H. Aoki, Rep. Prog. Phys. 50, 655 (1987).

[9] W. Florek, Rep. Math. Phys. 38, 235, 325 (1996).

[10] P.P. Divakaran, A.K. Rajagopal, Int. J. Mod. Phys. B 9, 261 (1995).

[11] W. Florek, Phys. Rev. B 55, 1449 (1997).

[12] W. Florek, S. Watcerz, J. Math. Phys. 39, 739 (1998).

[13] W. Florek, Acta Phys. Pol. A 92, 399 (1997).

[14] F. Ghaboussi, Int. J. Theor. Phys. 36. 923 (1997).

[15] A.O. Barut, R. Raczka, Theory of Group Representations and Applications, Polish Sci. Publ., Warsaw 1977.

[16] I. Dana, J. Zak, Phys. Rev. B 28, 811 (1983).

[17] J. Schwinger, Proc. Natl. Acad. Sci. (USA) 46, 570 (1960).

[18] A. Kerber, Algebraic Combinatorics via Finite Group Actions, BI Wissenschaftsverlag, Mannheim 1991, Sec. 1.5.

[19] N.B. Backhouse, C.J. Bradley, Quart. J. Math. Oxford 21, 203 (1970); ibid. 23, 225 (1972); N.B. Backhouse, ibid. 21, 277 (1970); ibid. 22, 277 (1971). 
[20] B.L. Davis, J. Phys. A, Math. Gen. 19, 831, 841, 847 (1986).

[21] G.W. Mackey, Acta Math. 99, 265 (1958).

[22] P.P. Divakaran, Rev. Math. Phys. 6, 167 (1994).

[23] M.S. Raghunathan, Rev. Math. Phys. 6, 207 (1994).

[24] S. Mac Lane, Homology, Springer-Verlag, Berlin 1963.

[25] P.P. Divakaran, A.K. Rajagopal, Physica C 176, 457 (1991).

[26] D.C. Tsui, H.L. Störmer, A.C. Gossard, Phys. Rev. Lett. 48, 1559 (1982).

[27] S. Das Sarma, A. Pinczuk, Perspectives in Quantum Hall Effect, Wiley, New York 1997.

[28] R. Shankar, G. Murthy, Phys. Rev. Lett. 79, 4437 (1997); G. Murthy, R. Shankar, in: Composite Fermions - A Unified View of the Quantum Hall Reyime, Ed. O. Heinonen, World Sci., New York 1998. 